\title{
La planeación pedagógico-didáctica implementada en el área de inglés en las comunas 1 y 2 de Bucaramanga, que atiende estudiantes en condición de vulnerabilidad en los niveles de básica y media: retos y oportunidades
}

\author{
Jorge Wilson Torres Hernández \\ María Cristina Gamboa Mora ${ }^{2}$
}

Recibido: 08-04-2020

Aceptado: 06-05-2020

\section{Resumen}

La política educativa colombiana implementada en el período 2002-2018 estableció acciones para la atención educativa de las poblaciones enmarcadas en los principios de inclusión y equidad, centrando su interés en las poblaciones en condiciones de vulnerabilidad, y desarrollando herramientas para fortalecer las prácticas escolares y mejorar los aprendizajes de la población. El objetivo de la investigación en la fase preliminar es caracterizar las unidades didácticas implementadas en el área de inglés en las comunas 1 y 2 de Bucaramanga, que atienden estudiantes en condición de vulnerabilidad en el nivel de básica y media. Para cumplir con el propósito se planteó una investigación de tipo exploratoria y descriptiva, centrada en el análisis del contenido de la legislación educativa colombiana para la definición de las categorías

1. Institución Educativa Dámaso Zapata, Bucaramanga, Colombia. Universidad de las Américas y el Caribe (UNAC), Colima, México.

Correo electrónico: jtorres@unac.edu.mx

ORCID: https://orcid.org/0000-0002-7944-0820

Google Scholar: https://scholar.google.com/citations?user=b4cqQ8wAAAAJ\&hl=es

2. Universidad Nacional Abierta y a Distancia (UNAD), Bogotá, Colombia. Docente categoría asociado, Líder del grupo de investigación AMECl.

Correo electrónico: maria.gamboa@unad.edu.co

ORCID: https://orcid.org/0000-0001-8459-099X

Google Scholar: https://scholar.google.es/citations?user=_180qYcAAAAJ\&hl=es 
emergentes de análisis; dichas categorías se emplearon para valorar las planeaciones pedagógico-didácticas de las instituciones. Se encontró que la planeación en el área de inglés proyectada para la población vulnerable se centra en la definición de la evaluación, y se descuidan aspectos relevantes del marco de referencia del Plan Nacional de Bilingüismo como: la inclusión de los derechos básicos de aprendizaje, la implementación del material didáctico y las funciones de la segunda lengua. Se concluye que la planeación es fundamental para articular adecuadamente el currículo pretendido - que se configura con base en la normatividad analizada-, el currículo aplicado - que se fundamenta en la planeación- y el currículo logrado - que corresponde al que manifiestan los estudiantes en las pruebas masivas de evaluación-, lo cual se hace necesario para la adquisición efectiva de una segunda lengua. Finalmente, con base en los resultados de la caracterización se plantean retos y oportunidades desde la planeación pedagógicodidáctica para el área, con el fin de encaminar las acciones al cumplimiento de los objetivos del Plan Nacional de Bilingüismo.

Palabras clave: planeación pedagógico-didáctica, currículo en inglés, Plan Nacional de Bilingüismo, vulnerabilidad.

\section{The pedagogical-didactic planning implemented in the English area in communes 1 and 2 of Bucaramanga, which serves students in vulnerable conditions at the basic and secondary levels: challenges and opportunities}

\section{Abstract}

The Colombian educational policy implemented in the period 20022018 established actions for the educational attention of populations framed in the principles of inclusion and equity, focusing its interest 
on populations in conditions of vulnerability, and developing tools to strengthen school practices and improve the learning of the population. The objective of the research in the preliminary phase is to characterize the didactic units implemented in the English area in communes 1 and 2 of Bucaramanga, which serve vulnerable students at the elementary and middle school level. To fulfill the purpose, an exploratory and descriptive research was proposed, focused on the analysis of the content of Colombian educational legislation for the definition of emerging categories of analysis; These categories were used to assess the pedagogical-didactic planning of the institutions. It was found that the planning in the area of English projected for the vulnerable population focuses on the definition of the evaluation, and relevant aspects of the frame of reference of the National Plan for Bilingualism are neglected, such as: the inclusion of basic learning rights, implementation of the teaching material and the functions of the second language. It is concluded that planning is essential to adequately articulate the intended curriculum - which is configured based on the regulations analyzed-, the applied curriculum — which is based on planningand the achieved curriculum - which corresponds to that expressed by students in the massive assessment tests-, which is necessary for the effective acquisition of a second language. Finally, based on the results of the characterization, challenges and opportunities arise from pedagogical-didactic planning for the area, in order to direct actions to fulfill the objectives of the National Plan for Bilingualism.

Keywords: pedagogical-didactic planning, curriculum in English, National Plan for Bilingualism, vulnerability. 


\section{Introducción}

En el año 2004 el Gobierno nacional en colaboración del British Council diseñan el Programa Nacional de Bilingüismo (PNB) para ser implementado a lo largo y ancho del territorio nacional y cuya meta radica en "lograr ciudadanos y ciudadanas capaces de comunicarse en inglés, de tal forma que se puedan insertar al país en los procesos de comunicación universal, en la economía global y en la apertura cultural, con estándares internacionalmente comparables" (Ministerio de Educación Nacional [MEN], 2006). En ese sentido Fandiño, Bermúdez y Lugo (2012), afirman que el PNB fue implementado no solo como un instrumento que pretende aportar a mejorar la calidad de la educación, sino que también fue concebido como una estrategia para la promoción de la competitividad. Asimismo, en cuanto al factor lingüístico, el MEN (2005) señala que a través del PNB se plantea dar respuesta a las necesidades nacionales en relación con el inglés, anhela formar docentes y estudiantes de educación básica, media y superior capaces de responder a un nuevo entorno bilingüe; de igual forma, se fomenta y ampara el manejo de otras lenguas en poblaciones étnicas, raizales y de frontera.

El PNB se ha fijado como meta que el $50 \%$ de los estudiantes alcancen el nivel B1 según el Marco Común Europeo de Referencia (MCER) para el año 2025, se han realizado acciones específicas hacia el alcance de la misma, por un lado, se ha efectuado la preparación de los docentes en sus competencias comunicativas al igual que la actualización en las metodologías recientes de enseñanza de L2 en menor medida, y se han realizado inmersiones en países angloparlantes, por otro lado, se llevó a cabo el desarrollo de un material didáctico en el año 2012 para la enseñanza en los grados de la educación media denominado "English Please!". Durante los primeros años del PNB se empieza a transformar el aprendizaje del inglés en los colegios públicos del país, no obstante, el MEN en el año 2014 evidencia que estas acciones no han tenido 
los resultados esperados en las pruebas Saber 11, los resultados de la aplicación son muy bajos en relación a los objetivos, un $51 \%$ de los alumnos tienen un nivel -A 1 , un $35 \%$ se encuentra en $A 1$, un $7 \%$ se encuentra en $A 2$ y tan solo un $7 \%$ se encuentra en B1 y B+. (Icfes, 2016).

\section{Referentes teóricos}

\section{La legislación colombiana frente a las necesidades de la adquisición de una segunda lengua}

El Ministerio de Educación Nacional observó la necesidad de constituir una propuesta metodológica en el área de idioma extranjero que coadyuvara a alcanzar aprendizajes reales de lengua extranjera (L2) con los niveles pretendidos, la cual previera oportunidades de aprendizaje para todos los estudiantes del territorio nacional. En ese sentido, en 2016 se constituyó el currículo sugerido de inglés para Colombia; esta propuesta analizó las mallas curriculares de 30 instituciones educativas del país teniendo en consideración las necesidades de la sociedad y las necesidades de la escuela. De igual manera, en estos ejes de referencia, el MEN (2016a) incluye los derechos básicos de aprendizaje en $\mathrm{L} 2$ en concordancia con los estándares básicos de competencias en lengua extranjeras. Estos derechos son herramientas que encaminan la toma de decisiones en relación a lo que cada niño debe aprender, cómo lo aprende y cómo se aborda, teniendo en cuenta las características de los estudiantes y su contexto. En esta línea, el currículo sugerido brinda apoyo en la construcción de mallas curriculares pertinentes en las instituciones educativas ya que implementa procesos de planeación significativa y contextualizada acorde a la vanguardia del proceso enseñanza-aprendizaje de L2. De la misma forma, un grupo representativo de instituciones educativas del país trabaja con la serie de libros Way to Go!, concebida para adaptarse a las necesidades particulares de las escuelas del país. Esta colección posee unas características específicas que han sido determinadas para 
asistir la integración de los libros en el sistema educativo público colombiano: 1) flexibilidad, 2) adaptabilidad, y 3) habilidades para el siglo XXI.

Con respecto al año 2017, luego de poner en acción las nuevas estrategias del PNB, los estudiantes que alcanzaron el nivel B1 y B+ en el área de inglés en la prueba Saber 11 son tan solo el $4.75 \%$ (Icfes, 2017). En otras palabras, estos datos muestran que no han sido significativos los alcances en cuanto al dominio del idioma extranjero en el nivel de básica y media, el cual supone alcanzar un nivel B1 o superior según el Marco Común Europeo de Referencia para las lenguas (Consejo de Europa, 2002)British Council, s. f.). Estos resultados reflejan que existe una brecha considerable entre el nivel actual de competencia en idioma extranjero y el nivel esperado por el PNB. Las distintas necesidades de los niños y niñas colombianas en relación con el aprendizaje de este idioma son un reto para la educación pública en Colombia. En este sentido, la Ley General de Educación colombiana (Ley 115 de 1994) sostiene que la educación cumple una función social consecuente con las necesidades e intereses de las personas, de la familia y de la sociedad; igualmente, es responsabilidad del Estado colombiano garantizar el acceso al servicio educativo púbico y de calidad. Al respecto, el Gobierno nacional a través del Plan Nacional de Desarrollo 20142018 considera "la educación como el más poderoso instrumento de igualdad social y crecimiento económico en largo plazo, con una visión orientada a cerrar brechas de acceso y calidad al sistema educativo" (DNP, 2015, p.77). En ese orden de ideas, el Ministerio de Educación Nacional colombiano asiste a las personas en situación de vulnerabilidad con recursos del Sistema General de Participaciones, de forma que se originen los recursos suficientes para la creación de proyectos que tengan como pilar fundamental estrategias de permanencia educativa de la población.

En relación con la política local, Rodolfo Hernández (2016) en el Plan de Desarrollo del Municipio de Bucaramanga 2016-2019, manifiesta que su propósito reside en la realización de una gestión pública municipal eficiente con base en su lema de gobierno "Lógica, Ética y Estética". De esta forma, toma como punto de partida 
la "Ética" para dirigir los recursos públicos hacia la asistencia y atención integral de grupos de población vulnerable, para alcanzar mínimos comunes de vida en equidad. En segundo lugar, la "Lógica", busca mejorar las condiciones de vida de grupos organizados de habitantes por medio de apoyo, acompañamiento y orientación; con ello se busca generar capacidades individuales y sociales que construyan y densifiquen el tejido social, para así construir una sociedad civil organizada. Por último, la "Estética" pretende reducir las barreras espaciales y culturales — tanto públicas como privadas-, que existen en la ciudad; esto tiene como objetivo lograr una comunidad de ciudadanos integrada por medio de la diversidad, en un espacio entendido como una sola ciudad, con doble vocación: rural y urbana. Con respecto a la enseñanza del bilingüismo, la Secretaría de Educación del municipio de Bucaramanga (SEB) trabaja con la Cámara de Comercio y el British Council en el Programa Municipal de Bilingüismo desde su creación en el año 2014. De acuerdo con el Plan de Desarrollo del Municipio de Bucaramanga 2016-2019 se pretende no solo mejorar la calidad de las políticas educativas, sino a su vez replantear los programas y generar estrategias para convertir a los alumnos y a los docentes en personas competitivas que los guíen hacia la búsqueda de nuevas oportunidades a través del crecimiento individual y colectivo. Este proyecto durante el año 2018 ha beneficiado a 600 docentes de primaria y secundaria y alrededor de 10000 alumnos de instituciones educativas públicas. No obstante, la secretaría de Educación del municipio no cuenta con proyectos que propendan mejorar la calidad del proceso de enseñanza-aprendizaje en el área de bilingüismo de las comunas 1 y 2 en el norte de la ciudad, incumpliendo con su propósito de brindar una atención prioritaria a las personas pertenecientes a la población vulnerable.

\section{El currículo y la planeación pedagógico-didáctica}

Robitalle y Garden (1996, citados en Gamboa, 2014) establecieron un modelo conceptual curricular que fundamenta la articulación entre el currículo pretendido, el aplicado y el logrado, que se con- 
cibe como un factor explicativo que subyace al rendimiento de los estudiantes. El currículo pretendido atiende al contexto educativo nacional con el propósito de suplir las necesidades educativas; en el caso descrito, el contexto corresponde a los estudiantes en condición de vulnerabilidad en el nivel de básica y media de las comunas uno y dos de Bucaramanga. El currículo aplicado corresponde a lo que se enseña en el aula que está estrechamente relacionado con la planeación pedagógico-didáctica e influenciado por diversos factores asociados a los actores educativos. El currículo logrado es aquel que se evidencia en los resultados que obtienen los estudiantes en las pruebas producto de sus aprendizajes y sus concepciones.

Por otra parte, la planeación didáctica involucra organización de objetivos, y actividades que dan sentido, significado y continuidad a un proceso educativo, permitiendo abordar, de manera ordenada y congruente, situaciones con sentido para la construcción de conocimiento (Ascencio, 2016). La planeación didáctica de los profesores es fundamental en la práctica docente en su dimensión ontológico-pedagógica, y una posibilidad de conocer el entramado (complexus) que impera en su diseño, creación y aplicación en el aula de clase; de ella depende el logro de una práctica eficiente encaminada al desarrollo de los aprendizajes esperados (García y Valencia-Martínez, 2014). Para complementar, y en correspondencia al mundo actual mediado por el uso de las tecnologías de la información y la comunicación (TIC), las estrategias pedagógico-didácticas debe ser innovadoras, y preferiblemente con la implementación de las TIC como herramienta motivadora para los estudiantes en formación. Al respecto la Organización de las Naciones Unidas para la Educación, la Ciencia y la Cultura, (Unesco, 2003, citado por Ospina, 2013) considera que las TIC en la educación han sido claves en la generación de habilidades intelectuales y sociales, motivación, colaboración, compromiso con el aprendizaje y un impacto en el rendimiento académico de los estudiantes. Cabero (2006, citado por Paredes y Arruda, 2012), señala que la motivación que propicia el uso de las TIC en la enseñanza está asociada a los procesos comunicativos puesto que la mediación promueve clases más interesantes, 
aumenta el intercambio de conocimientos y existe una gran facilidad de acceso a la información.

\section{Metodología}

Teniendo como contexto las instituciones educativas de las comunas 1 y 2 del municipio de Bucaramanga, se consideró necesario llevar a cabo un análisis hermenéutico a los planes de área o unidades didácticas de los establecimientos, con el fin de determinar si se está llevando al terreno de lo práctico lo estipulado por el Plan Nacional de Bilingüismo (PNB) y su marco de referencia.

La investigación se configura de tipo exploratorio y descriptivo, con el propósito de identificar las categorías de análisis para las unidades didácticas del área de inglés en las dimensiones fundantes para abordar e implementar estrategias de apropiación que generen aprendizajes significativos. Hernández, Fernández y Baptista (2014), señalan que los estudios exploratorios y descriptivos fundamentan nuevos procesos de investigación.

\section{Población y muestra}

Población: instituciones educativas de las comunas 1 y 2 de Bucaramanga que atienden estudiantes en condición de vulnerabilidad en el nivel de básica y media.

Muestra: 5 instituciones educativas públicas de las comunas 1 y 2 de Bucaramanga. Las instituciones seleccionadas son producto de un muestreo por conveniencia que corresponden a las instituciones que aceptaron hacer parte del estudio, en respuesta a una invitación formal de la Secretaria de Educación de Bucaramanga (SEB). Al respecto Otzen y Manterola (2017) señalan que el muestreo por conveniencia permite seleccionar casos accesibles al investigador 
por cercanía o aceptación de los sujetos o instituciones objeto de investigación.

\section{Método}

El método de investigación es la hermenéutica a través del análisis de textos. El uso del término hermenéutica se remonta a la incorporación del instrumento Perihermenais en la obra Organun de Aristóteles (384-382 a. de C.), para referirse al análisis de los juicios y las preposiciones para un pensar adecuado. La hermenéutica es una ciencia y un arte que tiene por objeto la interpretación para develar el sentido de los mensajes, favoreciendo la comprensión. Es considerada la disciplina de la interpretación, lo que hace que las obras interpretadas se vuelvan abiertas, en movimiento, que de acuerdo con la lectura toman significado (Arráez, Calles y Moreno de Tovar, 2006).

Como técnicas de análisis documental, se implementan cuatro fases: la de interpretación —en la cual se describe el plan-, los referentes conceptuales inmersos en la planeación, el análisis y la valoración de las categorías emergentes. Las categorías o dimensiones se constituyen en el producto de la acción racional para la comprensión e interpretación, convirtiéndose en los ejes para la comprensión e interpretación comunicativa; estas surgen de la competencia comunicativa del intérprete, que subyace del conocimiento en el tema y de la comprensión de este como requisito para la acción comunicativa con el documento (Habermas, 2000, citado por Rojas, 2011).

Las categorías de análisis emergen de la revisión documental de la legislación colombiana con respecto a los estándares básicos y los derechos de aprendizaje para el área de inglés. Además, se revisó el Plan Nacional de Bilingüismo, que tiene como propósito mejorar la competitividad de los estudiantes del territorio nacional, conduciéndoles al logro del nivel B1 (intermedio, de acuerdo con el MCER) (Consejo de Europa, 2002). El concepto de competencia se aborda como "el nuevo paradigma de la educación" (Tobón, Pi- 
mienta y García, 2010, p, 22), y según las consideraciones que desde la experiencia docente y la práctica investigativa se ponen en escena. Para el análisis de la información recolectada, se definieron las categorías emergentes descritas en la figura 1.

Figura 1. Categorías de análisis para las unidades didácticas en el área de inglés.

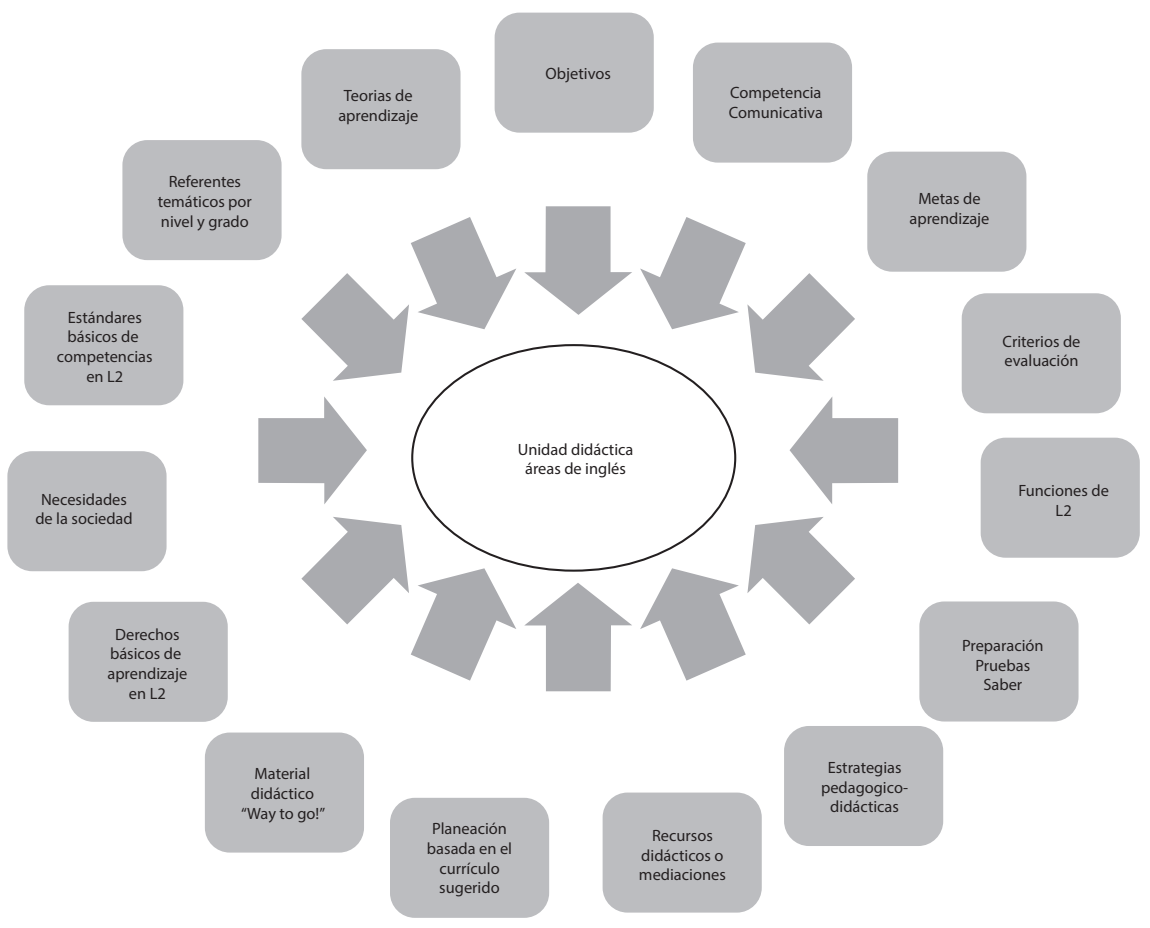

Fuente: elaboración propia.

\section{Análisis y discusión de los resultados}

Las unidades didácticas deben tener como propósito central guiar la construcción de las competencias relacionadas con el dominio del idioma inglés. 


\section{Descripción de las variables}

o categorías de análisis

1. Planeación basada en el currículo sugerido por el MEN (2016) El currículo sugerido (CS) (MEN, 2016b) propone para su implementación las siguientes tareas:

- Evaluar el Plan de área de inglés y de las mallas curriculares.

- Tomar decisiones sobre aspectos relevantes a adaptar de la propuesta a los documentos curriculares de la IE.

- Construir versiones revisadas de los documentos que articulen aspectos derivados de la propuesta curricular.

- Planear la puesta en escena del currículo en el aula.

\section{Material didáctico "Way to Go!"}

Los textos Way to Go! (2016) fueron desarrollados bajo el enfoque comunicativo y las metodologías de aprendizaje por tareas y proyectos, y se encuentran alineados con el currículo sugerido de inglés para grados de sexto $\left(6 .^{\circ}\right)$ a undécimo $\left(11 .^{\circ}\right)$ y con la Cartilla de los Derechos Básicos de Aprendizaje de Inglés. Es una serie de libros dirigida a los estudiantes y docentes de los grados sexto $\left(6 .^{\circ}\right)$, séptimo $\left(7 .^{\circ}\right)$ y octavo $\left(8^{\circ}{ }^{\circ}\right)$ de educación básica y media; son materiales enfocados en cuatro ejes temáticos transversales: Democracia y Paz, Salud, Medio Ambiente y Globalización

\section{Derechos básicos de aprendizaje (DBA) en $\mathbf{L 2}$}

Describen saberes y habilidades que los estudiantes deben aprender y desarrollar en el área de inglés en los grados de $6 .^{\circ}$ a $11 .^{\circ}$ del sistema educativo colombiano y se estructuran guardando coherencia con los Lineamientos Curriculares y los Estándares Básicos de Competencia (MEN, 2016a).

\section{Estándares básicos de competencias (EBC) en L2}

Criterios claros y públicos que permiten establecer cuáles son los niveles básicos de calidad a los que tienen derecho los niños y las 
niñas de todas las regiones de Colombia, para que puedan desenvolverse de manera efectiva (MEN, 2006).

\section{Necesidades de la sociedad}

Interpretaciones que la Constitución del 91 y el Ministerio de Educación han realizado de la sociedad colombiana y que la escuela debe contribuir a satisfacer (Chamorro, 2015, p. 7). En el contexto colombiano se refiere a la Ley 115 de 1994, el Plan Nacional Decenal de la Educación 2006-2016 (PNDE) (MEN, 2008), las metas educativas 2021 (OEI, 2010) y el Plan Nacional de Desarrollo 20142018 (DNP, 2015).

\section{Teoría de aprendizaje}

Se explica dentro del plan de área una teoría de aprendizaje que esté relacionada con la instrucción (Schunk, 2012).

\section{Objetivos}

Instrumentos didácticos que permiten racionalizar el proceso de enseñanza-aprendizaje en el aula, así como seleccionar las prácticas metodológicas y de evaluación coherentes con esos objetivos para dar cuenta de las necesidades de los estudiantes (Salcedo, 2011).

\section{Referentes temáticos por nivel y grado}

El plan de área propone habilidades y estrategias (cognitivas, metacognitivas, socioafectivas e interculturales), evidenciando el desarrollo de las capacidades de los estudiantes en la realización de tareas, desarrollo de proyectos y resolución de problemas (MEN, 2016c).

\section{Metas de aprendizaje}

Desarrollo de la competencia comunicativa en inglés, promoviendo y valorizando la colombiana (MEN, 2016b).

\section{Criterios de evaluación}

Articulación entre la evaluación del aprendizaje y la evaluación sumativa del proceso. La primera debe concretar la visión pragmática y holística del desarrollo de las competencias comunicativas 
que propone el currículo; la segunda, debe proporcionar criterios claros de medición de los desempeños alineados con los indicadores de logro y las estrategias instruccionales (Dochy, Moerkerke y Sluijsmans, 1999).

\section{Funciones de $L 2$}

Expresiones específicas sobre la comprensión del lenguaje y producción lingüística relacionadas con la cognición (Vivas, 2016).

\section{Competencia comunicativa}

Habilidad que tienen los sujetos de interpretar y representar diversos comportamientos sociales de una manera apropiada, dentro de una determinada comunidad de habla (Canale y Swain, 1980).

\section{Preparación pruebas Saber}

Inclusión de pruebas que sirven de preparación para el examen de Estado en grado $11 .^{\circ}$ en las mallas curriculares.

\section{Estrategias pedagógico-didácticas}

Técnicas, procedimientos, estrategias y métodos para mejorar el proceso de enseñanza de una manera más amplia, significativa y profunda (Medina, 2003).

\section{Recursos didácticos o mediaciones}

Materiales que ayudan a comunicar contenidos para el aprendizaje y pueden servir para estimular y dirigir el proceso de enseñanzaaprendizaje, total o parcialmente (Pérez, 2010).

El análisis hermenéutico de los planes de área de las cinco instituciones, se describe en las tablas 1 al 5. 
Tabla 1. Análisis hermenéutico al plan de área $n .^{\circ} 1$.

\section{Institución educativa pública $n .^{\circ} 1$}

Fase 1. Interpretación.

En el plan de área de esta Institución se aprecia una estructura basada en los estándares básicos de competencias - Lenguas extranjeras: inglés.

\section{Fase 2. Referentes.}

Para los grados de preescolar y primaria, solo se habla de una competencia: interpretativa. Para los grados de básica secundaria, se incrementa el horizonte de expectativas que puede percibirse del aprendiz. Se aclara que a pesar de algunos errores ortográficos, fonéticos y gramaticales cometidos, ya puede esperarse un vocabulario básico en inglés y el desarrollo de la expresión en cuanto a temas cotidianos o del "diario vivir". En esta fase se habla de dos competencias para los grados sexto y séptimo: interpretativa y argumentativa. Por su parte, para los grados octavo y noveno se incorpora, sumada a las dos anteriores, la competencia propositiva; en esta última, se busca una conexión entre las expectativas y posibilidades creadoras del estudiante anexadas a la proyección de manifestaciones ya expresadas en segunda lengua.

\section{Fase 3. Análisis.}

Existe una desarticulación entre algunos de los descriptores (justo después de indicar el grado escolar o nivel) y los indicadores de logro. Llama la atención, particularmente, en el caso de primaria, pues en los grados $4 .^{\circ}$ y $5 .^{\circ}$ se manifiesta que los educandos no tendrán un conocimiento más allá de su cotidianidad, a pesar de que los referentes temáticos versan sobre tópicos de culturas del mundo, gentilicios y otros países.

Pese a emplear una base teórica o pedagógica que hace hincapié en la motivación y el desequilibrio cognitivo como metas de avance cognitivo, la cultura del mundo debe también ser aproximada, más aun cuando la web revela al planeta como una "aldea global" (Reyes, 2001).

\begin{tabular}{|c|c|}
\hline \multicolumn{2}{|c|}{ Fase 4. Valoración. } \\
\hline Categoría & Valoración \\
\hline 1 & No se evidencia una planeación basada en el currículo sugerido por el MEN. \\
\hline 2 & Esta institución no hace parte de las IE focalizadas, por lo tanto no maneja el material \\
didáctico (MEN, 2016d).
\end{tabular}

Fuente: elaboración propia. 
Tabla 2. Análisis hermenéutico al plan de área $n .^{\circ} 2$.

\section{Institución educativa pública $\mathrm{n}^{\circ} 2$}

Fase 1. Interpretación.

El colegio en cuestión maneja la modalidad multigrado, que comprende un pequeño número de estudiantes por cada curso, pero todos estos grados trabajados en la misma jornada y en simultaneidad. Al tratarse de un sector rural (veredal) se maneja este tipo de enseñanza (Unesco, 2003).

\section{Fase 2. Referentes.}

Como características esenciales de esta planeación, se pueden destacar los siguientes aspectos:

- Los aspectos a evaluar son: núcleo temático (unidad), temas (contenidos), objetivos (habilidades o indicadores de logro) y desempeños (criterios de evaluación).

- Para el caso de Preescolar, las actividades se complementan con la presentación de imágenes.

- A partir del grado tercero, se incorporan conceptos de estructura gramatical (preguntas, rutinas, origen o nacionalidad).

\section{Fase 3. Análisis.}

En el análisis de esta planeación es preciso mencionar algunos aspectos particulares de su estructura: - Esta planeación no cuenta con un soporte teórico (al menos no uno explícito) que se enuncie en su creación y cuyo sustento epistemológico sea la base para su consolidación.

- En el desarrollo de este no se menciona el concepto de competencias. Tampoco se evidencian procesos, lo que hace pensar que el enfoque pedagógico de la institución educativa no se decante hacia lo globalizado, sino a su entorno.

- No se mencionan los alcances que se busca obtener a través del Programa Nacional de Bilingüismo (MEN, 2006).

\begin{tabular}{|c|c|}
\hline \multicolumn{2}{|r|}{ Fase 4. Valoración. } \\
\hline Categoría & Valoración \\
\hline 1 & Esta malla curricular carece de una puesta en práctica del CS. \\
\hline 2 & Está institución no hace uso del material didáctico Way to Go! \\
\hline 3 & No evidencia los saberes y habilidades que deben desarrollar los alumnos. \\
\hline 4 & $\begin{array}{c}\text { Esta planeación carece de descriptores donde se evidencien los DBA en lengua } \\
\text { extranjera. }\end{array}$ \\
\hline 5 & Este plan de área carece de un marco de referencia para su desarrollo. \\
\hline 6 & $\begin{array}{c}\text { No presenta el desarrollo de las cuatro habilidades del idioma extranjero e igualmente } \\
\text { no se evidencian estrategias de aprendizaje. }\end{array}$ \\
\hline 7 & Esta planeación carece de una teoría de aprendizaje. \\
\hline 8 & $\begin{array}{l}\text { Aunque existe un apartado denominado "Objetivos" no se evidencia la forma de guiar } \\
\text { el proceso de aprendizaje. }\end{array}$ \\
\hline 9 & En esta malla no se evidencia la finalidad del aprendizaje de la lengua extranjera. \\
\hline 10 & La evaluación del aprendizaje se hace a través de evaluaciones escritas y orales. \\
\hline 11 & $\begin{array}{l}\text { No cuenta con descriptores que traten sobre las funciones del idioma en cada uno de } \\
\text { los periodos de clase. }\end{array}$ \\
\hline 12 & No menciona la competencia comunicativa en su desarrollo. \\
\hline 13 & No manifiesta el desarrollo de pruebas Saber. \\
\hline 14 & $\begin{array}{c}\text { No cuenta con apartados que evidencien los procedimientos usados por los docentes } \\
\text { para mejorar el proceso de enseñanza-aprendizaje. }\end{array}$ \\
\hline 15 & $\begin{array}{l}\text { En este plan de área no se hace alusión a los recursos que facilitan el aprendizaje de } \\
\text { L2. }\end{array}$ \\
\hline
\end{tabular}

Fuente: elaboración propia. 
Tabla 3. Análisis hermenéutico al plan de área $n .^{\circ} 3$.

\section{Institución educativa pública $n .^{\circ} 3$}

Fase 1. Interpretación.

En el plan de área se aprecia el orden, la organización y el respeto de lo jurídico en cuanto a derechos y compromisos que la educación tiene para otorgar a sus educandos en la construcción del saber.

En la planeación se evidencia la importancia del seguimiento al proceso desde diversas perspectivas: además de la colombiana (que desde 2006 tiene convenio con el British Council), se incorpora además el MCER (Marco Común Europeo de Referencia) y la asociación ALTA (Association of Language Testers in Europe) (British Council, s. f.).

De acuerdo con las expectativas que se busca lograr en la Institución Educativa, se proyectan los niveles A1 y A2, siendo este último el de mayor alcance, al estar en grado undécimo. En cuanto a la competencia comunicativa, se exponen en tal componente tres subcompetencias: lingüística (que alude a todo el conocimiento gramatical, sintáctico y semántico en el campo de la estructura de la lengua), sociolingüística (que refiere a lo concerniente a las relaciones interpersonales, el proceso comunicativo a nivel cultural internacional) y pragmática (que además del uso de la escena práctica, los incorpora a lo largo de los niveles desde básica primaria, pasando por básica secundaria y finalizando en media).

Cabe destacar además, que en este plan de área, se enfatiza en la preparación para las pruebas Saber 11 , enfocando el fortalecimiento de la competencia interpretativa y lectora, en especial, en los grados $10 .^{\circ}$ y $11 .{ }^{\circ}$.

Fase 3. Análisis.

La planeación de la institución es organizada, estructurada y actualizada, de acuerdo con lo propuesto a nivel ministerial, a propósito del Programa Nacional de Bilingüismo. Falta presentar el tipo de estrategias metodológicas y de desarrollo didáctico que se afianzan en el aula con el objetivo de hacer andar tales nociones, en armonía con el entorno. En otras palabras, falta enunciar el cómo lograr los objetivos propuestos.

\section{Fase 4. Valoración.}

\begin{tabular}{|c|c|}
\hline Categoría & Valoración \\
\hline 1 & $\begin{array}{l}\text { A pesar de evidenciarse una planeación basada en el currículo sugerido, su expectativa } \\
\text { con relación al desarrollo de competencia en L2 es limitada. }\end{array}$ \\
\hline 2 & $\begin{array}{c}\text { En esta institución no se enseña el idioma extranjero a través del uso del material didáctico } \\
\text { propuesto por el MEN para secundaria. }\end{array}$ \\
\hline 3 & Este plan de estudios carece de un descriptor que manifieste los DBA en cada nivel. \\
\hline 4 & $\begin{array}{l}\text { Los estándares básicos de competencia en L2 de este plan de área apuntan a un nivel más } \\
\text { bajo que lo propuesto por el MEN. }\end{array}$ \\
\hline 5 & $\begin{array}{l}\text { Este plan de estudios manifiesta los aspectos jurídicos que rigen la enseñanza del idioma } \\
\text { extranjero en Colombia. }\end{array}$ \\
\hline 6 & $\begin{array}{l}\text { Aunque se evidencia que se trabajan las cuatro habilidades dentro del plan de área, no se } \\
\text { evidencia la forma para desarrollarlas. }\end{array}$ \\
\hline 7 & Se evidencia una visión constructivista del aprendizaje. \\
\hline 8 & $\begin{array}{l}\text { Se enuncian objetivos para alcanzar en cada periodo y grado; no obstante, no se menciona } \\
\text { de qué manera lograrlo. }\end{array}$ \\
\hline 9 & Se evidencia la meta de aprendizaje del idioma extranjero en este plan de área. \\
\hline 10 & $\begin{array}{l}\text { Se manifiestan los dos tipos de evaluación para el aprendizaje y evaluación del aprendizaje } \\
\text { en este plan de área. }\end{array}$ \\
\hline 11 & Este plan carece de la incorporación de las funciones de la lengua. \\
\hline 12 & Se evidencia las competencias comunicativas: lingüística, sociolingüística y pragmática. \\
\hline 13 & Se realiza una preparación para la prueba Saber 11 en los grados $10 .^{\circ}$ y $11 .^{\circ}$. \\
\hline 14 & Las estrategias metodológicas usadas son cognitivas y de memoria . \\
\hline 15 & cursos didácticos para la enseñanza del idioma extranjero. \\
\hline
\end{tabular}

Fuente: elaboración propia. 
Tabla 4. Análisis hermenéutico al plan de área $n .{ }^{\circ} 4$.

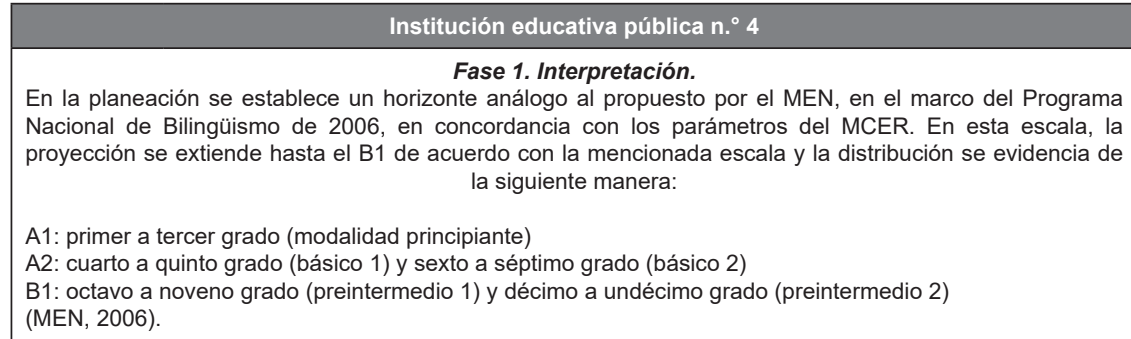

Fase 2. Referentes.

El plan de área expone que su modalidad es "Learning by doing" fundamentada en el hacer para aprender, lo cual le diferencia de otras planeaciones observadas en la zona norte de Bucaramanga. La estructura presenta los siguientes descriptores: período, objetos de mediación (referentes conceptuales), indicadores de desempeño, competencia del saber, competencia del hacer, competencia del ser-convivir y evidencias de aprendizaje.

Se describen actividades complementarias o "proyectos" que figuran al final de la planificación de cada grado o nivel, que bien pueden ser un baluarte adicional en la búsqueda de consolidación del aprendizaje de la segunda lengua. En la parte preliminar de esta planeación se efectúa además, una especie de proyección de competencias a manera de paralelo ubicando las que establece el MEN y en frente, las de la Institución Educativa La Juventud.

Fase 3. Análisis.

Es una planeación bien estructurada y organizada, se encuentra actualizada y propone unos aspectos interesantes no vistos en otros textos análogos de la zona norte de Bucaramanga.

\section{Fase 4. Valoración.}

Es una planeación bien estructurada y organizada, se encuentra actualizada y propone unos aspectos interesantes no vistos en otros textos análogos de la zona norte de Bucaramanga.

\begin{tabular}{|c|c|}
\hline Categoría & Valoración \\
\hline 1 & Esta planeación propone un horizonte análogo al descrito en el currículo sugerido. \\
\hline 2 & En esta institución educativa no se trabaja el libro Way to Go! \\
\hline 3 & Esta malla curricular no hace uso de los DBA para su desarrollo. \\
\hline 4 & $\begin{array}{l}\text { Se evidencia el uso de los estándares básicos de competencias en cada uno de los niveles } \\
\text { y periodos. }\end{array}$ \\
\hline 5 & Se manifiestan las necesidades de la sociedad en la justificación de su malla curricular. \\
\hline 6 & $\begin{array}{c}\text { Se manifiestan de forma organizada los objetivos en secuencia para cada grado y las } \\
\text { evidencias de aprendizaje. }\end{array}$ \\
\hline 7 & Se evidencia una visión constructivista del aprendizaje. \\
\hline 8 & $\begin{array}{l}\text { Este plan de área contiene descriptores de objetivos que guian el proceso enseñanza- } \\
\text { aprendizaje de L2. }\end{array}$ \\
\hline 9 & Se manifiesta la meta definida por el MEN para el aprendizaje del inglés en Colombia. \\
\hline 10 & $\begin{array}{l}\text { Este plan de área hace hincapié en los dos tipos de evaluación, denominándolos evidencias } \\
\text { de aprendizaje (sumativa y formativa). }\end{array}$ \\
\hline 11 & Se evidencian las funciones de la lengua plasmadas en el plan de área de esta institución. \\
\hline 12 & Este plan de área manifiesta las competencias a desarrollar en lengua extranjera. \\
\hline 13 & Este plan de área no muestra una preparación para la prueba Saber 11. \\
\hline 14 & $\begin{array}{l}\text { Se nombran estrategias metodológicas para mejorar el aprendizaje de la lengua; no } \\
\text { obstante, no se evidencia de qué manera se potencian, afianzan y dinamizan en la práctica. }\end{array}$ \\
\hline 15 & $\begin{array}{l}\text { Esta planeación curricular cuenta con descriptores donde se enuncia los materiales } \\
\text { didácticos usados para la enseñanza. }\end{array}$ \\
\hline
\end{tabular}

Fuente: elaboración propia. 
Tabla 5. Análisis hermenéutico al plan de área $n .{ }^{\circ} 5$.

\section{Institución educativa pública $\mathrm{n}^{\circ} 5$}

Fase 1. Interpretación.

Este plan de área fundamenta su estructura en los DBA y los EBC en inglés. Es un texto apropiado en términos de actualización y pertinencia.

\section{Fase 2. Referentes.}

Los aspectos propuestos en la planeación de la institución son:

- Estándar

- Competencia

- Logro

- Indicador

- Eje temático

- Metodología

- Recursos

- Evaluación

- Tiempo

- Actividades de refuerzo

La planeación se estructura a través de una "Secuencia didáctica" que divide el aprendizaje en tiempos o momentos.

\section{Fase 3. Análisis.}

Pese a poseer una estructura teórica, sugerida y epistemológica sólida, esta planeación podría tener en cuenta los siguientes aspectos:

- Dinamizar los procesos propuestos en el texto. No basta con proponerlos en el papel. Quizás un enriquecimiento en el cómo más allá de el qué haría interesante el ruedo de los procesos reflejados en el texto.

-Tener un sustento teórico al comienzo del Plan de Área con el fin de orientar su rumbo. En términos de aspectos estructurales y actualización es pertinente.

\section{Fase 4. Valoración.}

Con base en las categorías de análisis para la unidad didáctica o planeación de área.

\begin{tabular}{|c|c|}
\hline Categoría & Valoración \\
\hline 1 & $\begin{array}{c}\text { Esta planeación está basada el currículo sugerido y ha llevado a cabo las diferentes } \\
\text { tareas para su implementación en la IE. }\end{array}$ \\
\hline 2 & $\begin{array}{c}\text { La institución educativa, al ser parte de otra más grande, acoge las directrices que esta } \\
\text { plantea; en este caso se trabaja el material Way to Go! }\end{array}$ \\
\hline 3 & Esta malla curricular fundamenta su estructura en los DBA. \\
\hline 4 & $\begin{array}{l}\text { Se evidencia la fundamentación de los estándares básicos de competencias en L2 } \\
\text { incluidos en este plan de área. }\end{array}$ \\
\hline 5 & No se evidencian las necesidades de la sociedad en el desarrollo del plan de área. \\
\hline 6 & $\begin{array}{l}\text { El plan de área propone referentes temáticos acordes y secuenciales para el desarrollo } \\
\text { de habilidades y estrategias de L2. }\end{array}$ \\
\hline 7 & Se evidencia una visión sociocultural en el desarrollo del plan de área. \\
\hline 8 & Los objetivos planteados dan respuesta a las necesidades de los educandos. \\
\hline 9 & $\begin{array}{l}\text { La competencia comunicativa es concebida como la meta del aprendizaje de lengua } \\
\text { extranjera en esta malla curricular. }\end{array}$ \\
\hline 10 & $\begin{array}{l}\text { Este plan de área hace uso tanto de la evaluación del aprendizaje como de la } \\
\text { evaluación para el aprendizaje. }\end{array}$ \\
\hline 11 & $\begin{array}{l}\text { Este plan de área carece de un apartado donde se manifiesten las funciones de la } \\
\text { lengua en los diferentes niveles y periodos académicos. }\end{array}$ \\
\hline 12 & $\begin{array}{l}\text { Este plan de área propone el desarrollo de las tres competencias en L2: lingüística, } \\
\text { sociolingüística y pragmática. }\end{array}$ \\
\hline 13 & $\begin{array}{c}\text { Esta malla curricular incluye pruebas tipo Saber como evaluación acumulativa para } \\
\text { cada periodo y grado en secundaria. }\end{array}$ \\
\hline
\end{tabular}




\begin{tabular}{|c|c|}
\hline Categoría & Valoración \\
\hline 14 & $\begin{array}{r}\text { Se evidencia el uso de métodos de enseñanza para L2 como aprendizaje basado en } \\
\text { tareas y aprendizaje basado en proyectos. }\end{array}$ \\
\hline 15 & En esta planeación se encuentra un descriptor denominado recursos en el que se \\
describen las herramientas didácticas.
\end{tabular}

Fuente: elaboración propia.

Con respecto a las primeras seis categorías de análisis que corresponden a: referentes temáticos por nivel y grado, material didáctico Way to Go!, derechos básicos de aprendizaje en L2, estándares básicos de competencias en $L 2$, necesidades de la sociedad y referentes temáticos por nivel y grado, se obtiene que en las planeaciones pedagógico-didácticas del área de inglés:

- En un $80 \%$ de las instituciones educativas no se implementa el material didáctico Way to Go!, los derechos básicos de aprendizaje, ni se consideran las necesidades de la sociedad.

- En un $40 \%$ de las instituciones educativas se implementan los referentes temáticos por nivel y grado y los estándares básicos de competencias en L2. En la figura 2, se resume la valoración de las seis primeras categorías emergentes para el análisis de las unidades didácticas de las cinco instituciones participantes. 
Figura 2. Valoración de las seis primeras categorías emergentes de análisis para las cinco unidades didácticas o planes de área de las instituciones educativas.
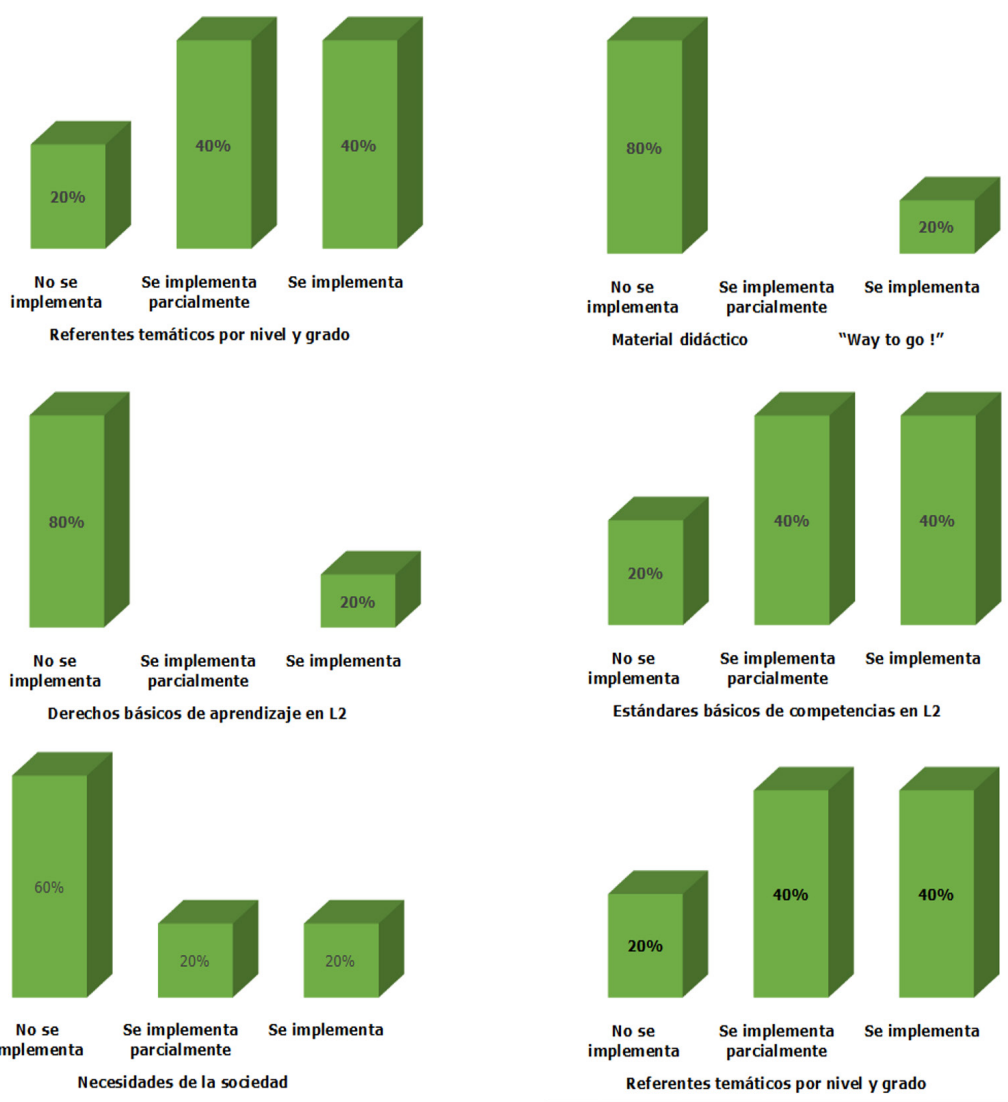

Material didáctico "Way to go !"

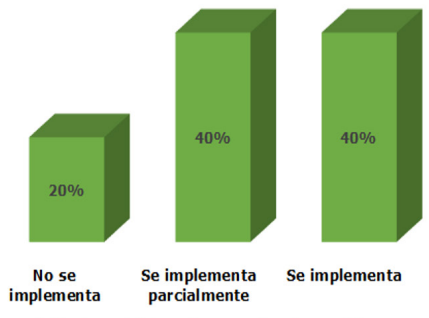

Estándares básicos de competencias en L2

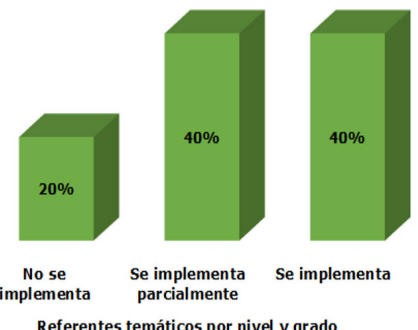

Referentes temáticos por nivel y grado

Fuente: elaboración propia.

- Sobre el análisis de las categorías 7-12 se encuentra que todas las instituciones plantean los criterios de evaluación para el área. En un $80 \%$ no se implementan las funciones de $\mathrm{L} 2$, en un $80 \%$ se implementan aspectos de la competencia comunicativa, y en un 60 $\%$ se implementan teorías y metas de aprendizaje. En la figura 3, se resume la valoración de las categorías 7-12 para el análisis de las unidades didácticas de las cinco instituciones participantes. 
Figura 3. Valoración de las categorías 7 al 12 para el análisis de las cinco unidades didácticas o planes de área de las instituciones educativas.
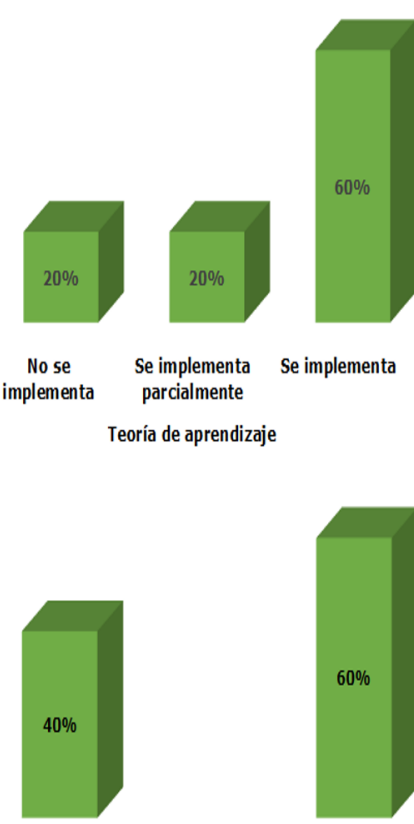

No se implementa Se implementa parcialmente

Metas de aprendizaje

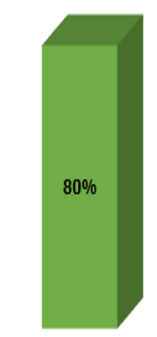

No se implement

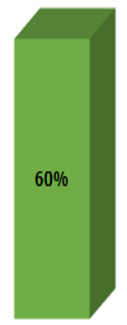

Se implementa

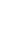

Se implementa parcialmente

Funciones de L2
No se implementa

Se implementa parcialmente

Criterios de evaluación

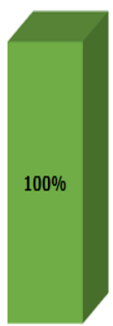

Se implementa

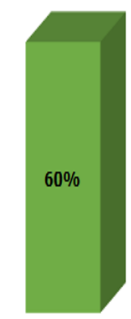

Se implementa

Objetivos 
pruebas Saber y las descripciones de los recursos para el área de inglés. En un $40 \%$ se describen las estrategias pedagógico-didácticas en las cuales se fundamentan los procesos educativos.

Figura 4. Valoración de las categorías 13 al 15 para el análisis de las cinco unidades didácticas o planes de área de las instituciones educativas.

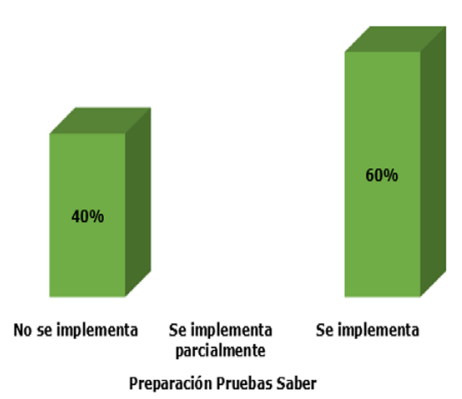

Preparación Pruebas Saber
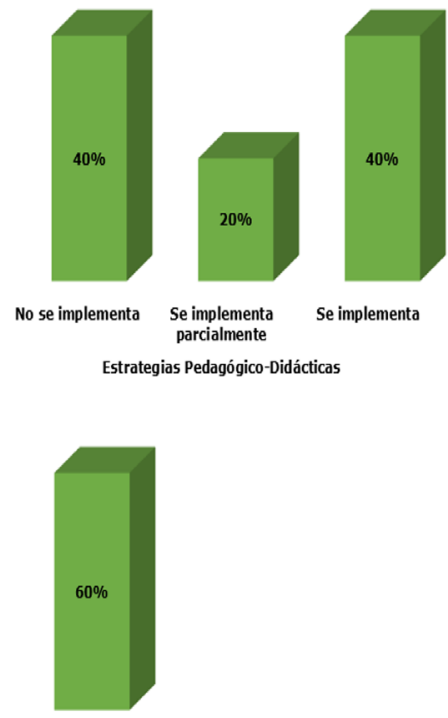

Se implementa

No se implementa Se implementa parcialmente

Fuente: elaboración propia.

Un aspecto que destaca en común en esta muestra de planeaciones es la búsqueda del fortalecimiento del vocabulario en las primeras etapas o niveles escolares, la relación del conocimiento con su entorno y cotidianidad y el abordaje de las cuatro habilidades del lenguaje (hablar, escribir, escuchar, leer). Como aspectos convergentes, se pueden destacar los siguientes:

- El concepto de lineamientos, estándares y demás sugerencias emanadas por el Ministerio de Educación, son reinterpretadas de formas diversas (a veces 
divergentes) por los planteles aquí analizados. Es un aspecto a tener en cuenta, si se habla de calidad educativa y garantías en el servicio.

- Los criterios establecidos por los planteles de las comunas 1 y 2 de Bucaramanga, son diversos; se evidencia que algunas instituciones educativas no describen claramente la orientación epistemológica que orienta su práctica.

- Por otra parte, no se desarrolla una segunda lengua con la simple idea de tener otro idioma en la reserva intelectual de la memoria. Hay una necesidad que, de hecho, está comprendida y estudiada en el marco de las garantías mínimas de aprendizaje que un estudiante debe percibir durante el desarrollo del plan de estudios. De ahí se deriva que, más que potenciar las cuatro habilidades comunicativas inherentes a cada idioma y en el caso del inglés (speaking, writing, listening y reading), se hace imperativa la implementación de competencias en el aula de clase. El modelo de competencias apoya el acercamiento y entrelazamiento de las instituciones educativas con la sociedad y sus dinámicas de cambio, con el fin de que estén en condiciones de contribuir tanto al desarrollo social y económico como al equilibrio ambiental y ecológico (Tobón et al., 2010). Una idea ulterior, a manera de reflexión de este compendio, se podría sugerir el empleo de un enfoque socioformativo (Tobón et al., 2010), en el caso de la dinamización de los planes de área llevados a la escena práctica del aula. Mejor aún, y teniendo en cuenta que actualmente se cuenta con profesores muy calificados para la enseñanza de la lengua extranjera, además de la proyección esperada desde la esfera gubernamental. ¿Qué pasaría si el desarrollo y dinamización de estas didácticas estuviese mediado por un trabajo elaborado de forma reflexiva, mediante una guía que acompañe el proceso? 


\section{Finalmente, en la tabla 6 se desglosan los retos y oportunidades que se definen con base en el análisis.}

Tabla 6. Retos y oportunidades para la enseñanza-aprendizaje del inglés como segunda lengua.

\begin{tabular}{|c|c|}
\hline Retos & Oportunidades \\
\hline $\begin{array}{l}\text { Articular las estrategias pedagógico-didácticas } \\
\text { para la promoción del desarrollo humano integral. }\end{array}$ & $\begin{array}{c}\text { Presentar de manera clara los objetivos, métodos } \\
\text { y criterios de evaluación }\end{array}$ \\
\hline $\begin{array}{l}\text { Promover una docencia de calidad basada en } \\
\text { la organización de los contenidos, metodologías } \\
\text { vanguardistas articuladas con las TIC. }\end{array}$ & $\begin{array}{l}\text { Sistematizar los marcos teóricos que } \\
\text { fundamentan el conocimiento disciplinar. }\end{array}$ \\
\hline $\begin{array}{l}\text { Implementar clases con intenciones claras para } \\
\text { la adquisición de habilidades y competencias } \\
\text { definidas por el MEN para el logro de los } \\
\text { objetivos de una Colombia bilingüe. }\end{array}$ & $\begin{array}{l}\text { Disminuir las improvisaciones en el aula, } \\
\text { garantizando sesiones de clase intencionadas. }\end{array}$ \\
\hline $\begin{array}{c}\text { Proyectar compromiso con la adquisición de una } \\
\text { segunda lengua, a partir de una planificación } \\
\text { adecuada y consensuada por los docentes de } \\
\text { área. }\end{array}$ & $\begin{array}{c}\text { Sistematizar las mejores experiencias educativas } \\
\text { para transferirlas a otros espacios educativos con } \\
\text { necesidades similares. }\end{array}$ \\
\hline $\begin{array}{l}\text { Promover las estrategias pedagógico-didácticas } \\
\text { exitosas en la adquisición del inglés. }\end{array}$ & $\begin{array}{c}\text { Aumentar el conocimiento sobre la efectividad } \\
\text { de las estrategias pedagógico-didácticas para el } \\
\text { aprendizaje del inglés. }\end{array}$ \\
\hline $\begin{array}{c}\text { Documentar las experiencias en el aula para } \\
\text { buscar el sentido y significado de ellas en } \\
\text { procura de un aprendizaje significativo de un } \\
\text { segundo idioma. }\end{array}$ & $\begin{array}{l}\text { Reflexionar sobre las decisiones tomadas y sus } \\
\text { implicaciones en el aprendizaje del inglés. }\end{array}$ \\
\hline $\begin{array}{c}\text { Promover las competencias docentes a través de } \\
\text { una organización de la planeación pedagógico- } \\
\text { didáctica del área. }\end{array}$ & $\begin{array}{c}\text { Formar al docente del área de inglés en } \\
\text { planeación pedagógico-didáctica para promover } \\
\text { aprendizajes significativos. }\end{array}$ \\
\hline $\begin{array}{l}\text { Lograr la certificación internacional de los } \\
\text { docentes en ejercicio en el nivel B2 o superior, } \\
\text { para garantizar aprendizajes significativos. }\end{array}$ & $\begin{array}{c}\text { Participar en los programas de capacitación } \\
\text { sobre formación en inglés y sus metodologías de } \\
\text { enseñanza que ofrece el MEN. }\end{array}$ \\
\hline $\begin{array}{l}\text { Asignar docentes calificados en el ár } \\
\text { para todos los niveles educativos de }\end{array}$ & $\begin{array}{l}\text { Mejorar la formación inicial de docentes del área } \\
\text { de inglés, a través de currículos pertinentes. }\end{array}$ \\
\hline
\end{tabular}

Fuente: elaboración propia.

\section{Conclusiones}

El Ministerio de Educación Nacional, a través del Programa Nacional de Bilingüismo bajo la tutela del British Council, ha sido un actor principal que ha fortalecido el aprendizaje del idioma extranjero en los colegios oficiales del país, reflejando una mejoría en los resultados de la prueba de Estado Saber 11 durante los últimos años. 
La valoración de las unidades didácticas permite reconocer aspectos del currículo pretendido y, a su vez, tener un panorama del currículo aplicado. Se trata de un insumo relevante para proponer mejoras desde la planeación pedagógico-didáctica, que lleven a nuevas propuestas de intervención didáctica con el propósito de valorar los resultados académicos de los estudiantes en el área de inglés.

La planeación pedagógico-didáctica es fundamental para articular adecuadamente el currículo pretendido (que se configura con base en la normatividad analizada), el currículo aplicado y el currículo logrado (que corresponde al que manifiestan los estudiantes en las pruebas masivas de evaluación). Ello se hace necesario para la adquisición efectiva de una segunda lengua.

\section{Para la reflexión}

Hay un sinnúmero de factores socioeconómicos y ambientales que promueven la mudanza o cambio frecuente de residencia por parte de las familias que habitan la zona norte de Bucaramanga. Una mirada reflexiva al respecto deja al descubierto el siguiente interrogante: ¿Cómo garantizar la calidad cognitiva manifestada en competencias de la lengua extranjera, para aquellos estudiantes que se cambian de domicilio y por ende de IE? Este interrogante, a manera de reflexión, invita a pensar en la necesidad de gestar no solo una "carta de navegación" más unificada, sino además, un constructo que involucre uno de los elementos más complejos a la hora de generar interés por el aprendizaje: la motivación de los aprendices. 


\section{Referencias bibliográficas}

Arráez, M., Calles, J. y Moreno de Tovar, L. (2006). La hermenéutica: una actividad interpretativa. Sapiens Revista Universitaria de Investigación, 7(2), 171-181. Recuperado de http://www.redalyc.org/ pdf/410/41070212.pdf

Ascencio, C. (2016). Adecuación de la planeación didáctica como herramienta docente en un modelo universitario orientado al aprendizaje. REICE. Revista Iberoamericana sobre Calidad, Eficacia y Cambio en Educación, 14(3), 109-130. Recuperado de http://www. redalyc. org/articulo.oa? $\mathrm{id}=55146042006$

Canale, M. y Swain, M. (1980). Theoretical Bases of Communicative Approaches to Second Language Teaching and Testing. Applied Linguistics, 1(1), 1-47.

Chamorro, D. (2015). Orientaciones para la evaluación curricular [sin publicar]. Barranquilla: Universidad del Norte.

Congreso de Colombia. (8 de febrero de 1994). Ley 115. Por la cual se expide la ley general de educación. Diario Oficial n. 41214. Recuperado de http://www.secretariasenado.gov.co/senado/basedoc/ ley_0115_1994.html

Consejo de Europa (2002). Marco común europeo de referencia para las lenguas: aprendizaje, enseñanza y evaluación. Madrid: MCED y Anaya.

DNP (Departamento Nacional de Planeación). (2015). Plan Nacional de Desarrollo 2014-2018. Todos por un nuevo país. Recuperado de https:// colaboracion.dnp.gov.co/CDT/PND/PND\%202014-2018\%20Tomo\%20 1\%20internet.pdf

Dochy, F., Moerkerke, G. y Sluijsmans, D. (1999). The use of self-, peer-, co-assessment in higher education: A review. Studies in Higher Education, 24(3), 331-350. 
Fandiño, Y., Bermúdez, J. y Lugo, V. (2012). Retos del Programa Nacional de Bilingüismo. Colombia Bilingüe. Educación y Educadores, 15(3), 363381. Recuperado de http://educacionyeducadores.unisabana.edu.co/ index.php/eye/article/view/2172/2912

Gamboa, M. (2014). La evaluación externa en el área de ciencias a través de las pruebas masivas a gran escala PISA y TIMSS. Análisis del desempeño de estudiantes colombianos y españoles a través de la comparación. Bogotá: Universidad Distrital Francisco José de Caldas; Universidad Nacional Abierta y a Distancia. Recuperado de https://stadium.unad.edu. co/preview/UNAD.php?url=/bitstream/10596/2792/4/9789588832692. pdf

García, M. y Valencia-Martínez, M. (2014). Nociones y prácticas de la planeación didáctica desde el enfoque por competencias de los formadores de docentes. Ra Ximhai, 10(5), 15-24. Recuperado de http:// www.redalyc.org/pdf/461/46132134001.pdf

Hernández, R. (2016). Plan de desarrollo 2016-2019. Gobierno de las ciudadanas y ciudadanos. Bucaramanga: Alcaldía de Bucaramanga. Recuperado de http://www.concejodebucaramanga.gov.co/ proyectos2016/PROYECTO_DE_ACUERDO_013.pdf

Hernández, R., Fernández, C. y Baptista, P. (2014). Metodología de la investigación (6. ed.). Ciudad de México: McGraw Hill.

Icfes (Insitituto Colombiano para el Fomento de la Educación Superior). (2016). Informe nacional de resultados Saber 11 2012-2015. Recuperado de http://www.icfes.gov.co/docman/investigadores-y-estudiantes-deposgrado/informes-de-resultados- evaluaciones-nacionales/informesde-saber-pro/2684-informe-nacional-de-resultados-saber-pro-20122015/file?force-download=1

https://www.icfes.gov.co/documents/20143/193784/Informe\%20 nacional\%20saber\%2011\%202015.pdf

Icfes (Insitituto Colombiano para el Fomento de la Educación Superior). (2017). Informe nacional. Resultados 2014-2 - 2017-2. Saber 11. Bogotá: Ministerio de Educación Nacional; Icfes. Recuperado de https://www. 
icfes.gov.co/documents/20143/193784/Informe\%20nacional\%20de\%20 resultados\%20del\%20examen\%20saber\%2011\%20-\%202014-2\%20-\%20 2016-2.pdf

Medina, A. (2003). La didáctica: disciplina pedagógica aplicada. En A. Medina y F. Salvador (coords.), Didáctica general (pp. 3-37). Madrid: Prentice Hall.

MEN (Ministerio de Educación Nacional). (2005). Bases para una nación bilingüe y competitiva. Altablero, 37. Recuperado de http://www. mineducacion.gov.co/1621/article-97498.html

MEN (Ministerio de Educación Nacional). (2006). Estándares Básicos de Competencias en Lenguas Extranjeras: inglés. Formar en lenguas extranjeras: iel reto! Lo que necesitamos saber y saber hacer. Bogotá: Imprenta Nacional. Recuperado de https://redes.colombiaaprende. edu.co/ntg/men/archivos/Referentes_Calidad/Estandares_basicos_ de_competencias/Estandares_Basicos_Competencia_en_Lenguas_ Extranjeras_\%20Ingles.pdf

MEN (Ministerio de Educación Nacional). (2008). Plan decenal de educación 2006-2016. Compendio general. Pacto social por la educación. Recuperado de http://www.plandecenal.edu.co/cms/media/ herramientas/pnde_2006_2016_compendio.pdf

MEN (Ministerio de Educación Nacional). (2016a). Derechos básicos de aprendizaje: inglés. Grados 60 a 11‥ Bogotá: MEN. Recuperado de http://aprende.colombiaaprende.edu.co/ckfinder/userfiles/files/ cartillaDBA.pdf

MEN (Ministerio de Educación Nacional). (2016b). Diseñando una propuesta de currículo sugerido de inglés para Colombia. Bogotá: MEN; Colombia Bilingüe. Recuperado de https://aprende. colombiaaprende.edu.co/sites/default/files/naspublic/Anexo\%20 16\%20Dise\%C3\%B10\%20Propuesta\%20\%20Curriculo\%20Sugerido.pdf

MEN (Ministerio de Educación Nacional). (2016c). Orientaciones y principios pedagógicos. Currículo sugerido de inglés. Grados $6^{\circ}$ a $11^{\circ}$. 
English for Diversity and Equity. Bogotá: MEN; Colombia Bilingüe. Recuperado de https://aprende.colombiaaprende.edu.co/sites/default/ files/naspublic/Anexo\%2014\%200rientaciones\%20y\%20principios\%20 Pedagogicos.pdf

MEN (Ministerio de Educación Nacional). (2016d). Colección Way to Go! Recuperado de http://aprende.colombiaaprende.edu.co/ colombiabilingue/94010

OEI (Organización de Estados Iberoamericanos). (2010). Metas educativas 2021: la educación que queremos para la generación de los bicentenarios. Documento final. Madrid: Cepal; OEI. Recuperado de https://www.oei.es/Educacion/metas2021/documento-final

Ospina, C. (2013). Las TIC como herramienta de motivación en el aula [en línea]. Recuperado de http://intellectum.unisabana.edu.co/bitstream/ handle/10818/5358/129394. pdf?sequence=1\&isAllowed=y

Otzen, T. y Manterola, C. (2017). Técnicas de muestreo sobre una población a estudio. International Journal of Morphology, 35(1), 227232. Recuperado de https://scielo.conicyt.cl/pdf/ijmorphol/v35n1/ art37.pdf

Paredes, J. y Arruda, R. (2012). La motivación del uso de las TIC en la formación de profesorado en educación ambiental. Revista Ciência \& Educação, 18(2), 353-368. Recuperado de http://www.scielo.br/pdf/ ciedu/v18n2/a08v18n2.pdf

Pérez, S. (2010). Los recursos didácticos. Temas para la Educación. Revista Digital para Profesionales de la Enseñanza, 9. Recuperado de https:// www.feandalucia.ccoo.es/indcontei. aspx $\mathrm{d}=5156 \& \mathrm{~s}=5 \&$ in $d=231$

Reyes, G. (2001). Teoría de la globalización: bases fundamentales. Nómadas, 3. Recuperado de http://www.redalyc.org/pdf/181/18100308. pdf

Rojas, I. (2011). Hermenéutica para las técnicas cualitativas de investigación en ciencias sociales: una propuesta. Espacios 
Públicos, 14(31), 176-189. Recuperado de http://www.redalyc.org/ pdf/676/67621192010.pdf

Salcedo, H. (2011). Los objetivos y su importancia para el proceso de enseñanza-aprendizaje. Revista de Pedagogía, XXXII(91), 113-130. Recuperado de http://www.redalyc.org/pdf/659/65926549007.pdf

Schunk, D. (2012). Teorías del aprendizaje. Una perspectiva educativa (6. $a$ ed.). Greensboro: The University of North Carolina at Greensboro.

Tobón, S., Pimienta, J. y García, J. (2010). Secuencias didácticas: aprendizaje y evaluación de competencias. Ciudad de México: Pearson.

Unesco. (2003). Escuelas multigrados ¿Cómo funcionan? Reflexión a partir de la experiencia evaluativa del proyecto Escuelas Multigrado Innovadas [elaborado por T. Vargas; serie Cuadernos de Educación Básica para Todos] Arroyo Hondo: Unesco. Recuperado de http://unesdoc. unesco.org/images/0013/001374/137497so.pdf

Vivas, J. (2016). ¿'Por qué el estudio del lenguaje es fundamental para la cognición? Sophia. Colección de Filosofía de la Educación, 20, 67-85. Recuperado de http://www.redalyc.org/pdf/4418/441846839002.pdf

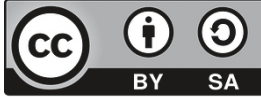

